\title{
TEMPORAL AND SALINITY IMPACTS ON THE MICROBIAL DIVERSITY AT THE EILAT, ISRAEL SOLAR SALT PLANT.
}

\author{
C.D. LITCHFIELD ${ }^{1, *}$ \\ A. OREN ${ }^{2}$ \\ A. IRBY $Y^{1,3}$ \\ M. SIKAROODI ${ }^{1}$ \\ P.M. GILLEVET ${ }^{1}$
}

Received: 19/08/08

Accepted: 20/10/08

\author{
${ }^{1}$ Department of Environmental Science \& Policy, \\ George Mason University, Manassas, Virginia, USA \\ ${ }^{2}$ Institute of Life Sciences, The Hebrew University \\ of Jerusalem, Israel \\ ${ }^{3}$ American Type Culture Collection, Manassas, Virginia, USA
}

*to whom all correspondence should be addressed: e-mail: clitchfi@gmu.edu

\begin{abstract}
A major solar salt works in Israel is located just north of the town of Eilat and close to the airport. This solar salt works has been in operation since 1977 and produces about 170,000 tons a year. A three-year study was initiated to examine the temporal and salinity impacts on the microbial community throughout this saltern. At the time this study was begun in the late 1990 's, there were several paradigms about the microbial community:

1. there is greatly reduced diversity as the water reaches saturation;

2. because of the relative stability of the salt concentrations in the various pans, there is little change in the microbial community; and,

3. halophilic Archaea are not found in ponds with less than $15 \%$ salt and halophilic Bacteria would not be found in pans at saturation.

The first paradigm was developed based on a few studies using one medium and single observations of various salterns. The second paradigm was an assumption that had not been systematically tested, and the third paradigm, at least for the Bacteria, was shown in 1980 not to be true with the discovery of the genus Halomonas.

We examined these paradigms by systematically studying on a seasonal basis the saltern at Eilat, Israel, as well as the Cargill Solar Salt Plant in Newark, California. Both traditional and molecular techniques have been used throughout these studies.

Several different approaches were taken: plate counts were made on several different media, whole community carbon requirements were determined, molecular fingerprinting of the whole microbial community using the amplicon length heterogeneity approach was performed to determine the changes in the community composition over time, and partial characterization of the isolated pure cultures were all performed. Samples were taken at least twice a year corresponding to the cooler and hotter times of the year.

Based on this extensive study there were significant differences in the plate counts and in the ratios of the various peaks found during the fingerprinting. It is safe to say now that the microbial community in the waters of a solar saltern is variable and both domains can be found throughout the saltworks.
\end{abstract}

KEYWORDS: bacteria, fingerprinting, community structure, lipid analysis, carbon sources, extracellular enzymes.

\section{BACKGROUND}

The Israel Salt Industries Ltd. solar salt works is located just north of the town of Eilat, Israel and close to the airport. This solar salt works has been in operation since 1980 and produces about 150,000 tons a year. A three-year study was initiated to examine the temporal and salinity impacts on the microbial community throughout this saltern. At the time this study was begun there were several paradigms about the microbial community:

1. there was little microbial diversity as the brine reached saturation; 
2. because of the relative stability of the salt concentrations in the various pans, there was little change in the microbial community; and,

3. halophilic Archaea would not be found in ponds with less than $15 \%$ salt and halophilic Bacteria would not be found in pans at saturation.

The first paradigm developed based on a few studies using one medium and single examinations of the salterns. The second paradigm was an assumption that had not been systematically tested, and the third paradigm, at least for the Bacteria, had been shown in 1980 not to be true with the discovery of the genus Halomonas (Vreeland et al., 1980).

There are three major groups of organisms in brines containing more than $12 \%$ salt. These include the halophilic (salt loving) members of the domain Bacteria which generally have a broad salt tolerance and the halophilic Archaea, the salt-loving bacteria, with a requirement for highly elevated concentrations of salt, often up to 10 times the salinity of seawater.

The purpose of this present study was to test the paradigm that because the salinity doesn't change significantly in the various pans, the microbial community will be very stable. The corollary to that was that halophilic Bacteria will not be found at salinities greater than $20 \%$, and halophilic Archaea will not be found at salinities less that $15 \%$ with respect to sodium chloride. Thus the overall aim is to determine the diversity within a solar saltern and demonstrate that these man-made ecosystems contain untapped biodiversity.

\section{MATERIALS \& METHODS Sample collection}

At the time of this study, the inlet waters were seawater from the Gulf of Aquaba and refuse brine from the desalination plant of Eilat. The brine flowed into a series of ponds during which it became more saturated with respect to salt. After sodium chloride precipitated the salt was recovered and another flooding of the crystallizers occurred.

Three to four liter samples were obtained from several locations within the Eilat, Israel solar salt work (Figure 1).

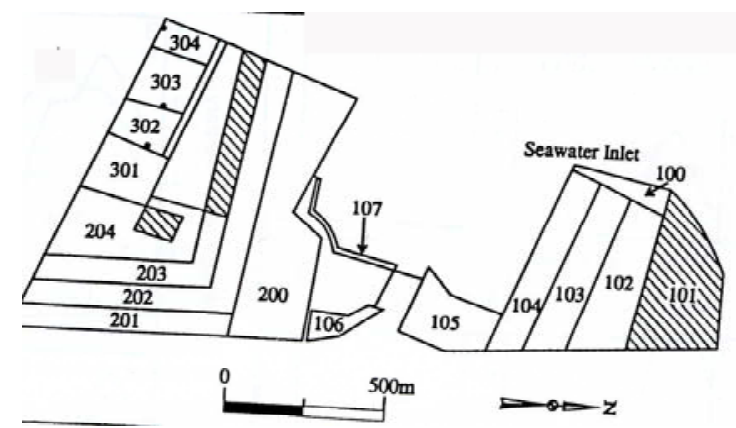

Figure 1. Plan of the Israel Salt Industries Ltd., Eilat, Israel, saltern

\section{Enumerations}

Two different media were used throughout this study. The first was a modified casamino acid medium made with tap water (MCAT) (Litchfield et al., 2005), and the second was a modification of the R2A medium (Difco). R2A was modified by the addition of $20 \mathrm{~g}$ magnesium sulfate per liter. Each medium was prepared to contain 4, 10, 20, and 25\% solar salt (w/v) and $\mathrm{pH} 7.3$.

Direct microscopic counts employed the method of Zweiful and Hägstrom (1995). This method eliminates intact dead cells by subjecting the preparation to a toluene washing step. Intact membranes retain the DAPI dye while injured/dead cells loose the dye.

\section{Metabolic diversity}

The metabolic potential of the whole microbial community was tested for ponds containing less than 14\% salt (Litchfield et al., 2001). Approximately 145 ul of the saline water was placed into each of the 96 wells in a BIOLOG GN1 plate. All plates were inoculated in triplicate and incubated at close to in situ temperatures. The plates were read daily for the first 
four days and weekly thereafter until there were no additional color changes. A positive carbon or nitrogen source was indicated by a change from colorless to pink or purple.

\section{Extracellular enzyme analyses}

Cultures were tested for extracellular enzyme production by streaking them onto plates containing casein (proteinase), DNA (DNAase), starch (amylase), olive oil (lipase). Zones of clearing or reactions with iodine to show hydrolysis of starch to simpler sugars were used to identify enzymatic activity. Gelatinase activity was detected by spotting the culture onto photographic film and observation of pittingas the gelatin layer was dissolved.

\section{Lipid analysis}

The lipid composition of the whole community was detected by Thin-Layer Chromatography as described previously (Litchfield and Oren, 2001).

\section{Molecular analyses}

The amplicon length heterogeneity (AL) procedure of Suzuki et al. (1998) was used. Briefly approximately four liters of water were centrifuged and the 16S rRNA gene was extracted from the resulting pellet with a Fast DNA Spin Kit for Soil (BIO 101, Vista CA). Following polymerase chain reaction (PCR) (Litchfield et al., 2006) with 5'-6-FAM-tagged forward primers for the Bacteria and the Archaea, the products were separated on a 96-glass capillary SpectruMedix Sequencer which measures the fluoresence for each amplicon or operational taxonomic unit (OTU). Peak height is proportional to the concentration of the OTU.

\section{RESULTS \\ Enumerations}

Table 1 shows the changes in the total colony -forming units (CFU) per $\mathrm{mL}$ and the total pigmented colony-forming units (TPCFU) per $\mathrm{mL}$ during the course of this study. There were essentially no pigmented colonies when the inlet was sampled, but this number increased with increasing salinity to where TCFU and TPCFU were essentially equal in the saturated salt pond (304). It is also noteworthy that the January 1997 samples were three to four orders of magnitude lower in their counts of microbial life on the MCAT medium. This despite the direct counts showing from nondetectable (pond 203) to $5 \times 10^{7}$ bacterial cells per $\mathrm{mL}$. The R2A medium resulted in consistently higher colony counts, but none quite reached the level of the direct counts. Thus, despite using a number of different media and different salt concentration, there is a large uncultivated microbial biomass existing in these ponds.

\section{Metabolic diversity}

Analysis of the BIOLOG data used the niche overlap procedure of Wilson and Lindow (1994). The inlet samples had 59 to 81, out of 95 potential nutrient sources, with overlapping dependent on the sampling period. This dropped to fewer than $20 \mathrm{common}$ carbon/nitrogen sources for the ponds containing $12-15 \%$ salt. There were no carbon sources that were commonly used regardless of location or season. This implies that in this system, the metabolic diversity decreases as the salinity increases. This results in a selection for microbes either able to grow at the elevated salt concentrations or those which have a requirement for higher salt concentrations. This is unlike other salterns were at least nine to ten carbon sources were routinely metabolized (Litchfield et al., 2001).

\section{Lipid patterns}

Variability was also observed here, but phosphatidy glycerol and phosphatidyl glycerol sulfate were encountered routinely (data not shown).

\section{Enzymatic assays}

Ninety pure cultures obtained during the course of this study were screened for the production of extracellular enzymes. The data (Figure 2), show that DNAse and amylase activity are the two most common extracellular enzyes regardless of the source of the cultures with gelatinase the next most frequently noted extracellular enzyme. 


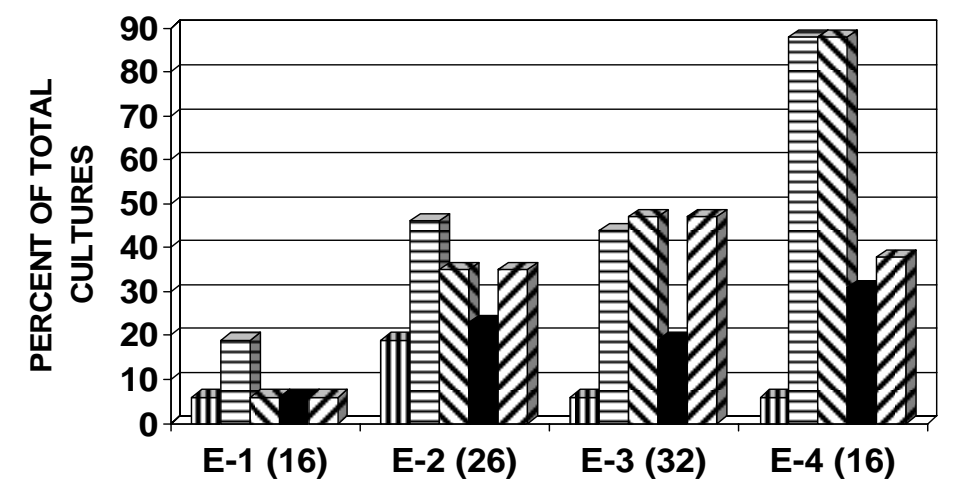

II Lipase $\boxminus$ DNAse $\mathbf{D}$ Amylase $\square$ Proteinase $Z$ Gelatinase

Figure 2. Extracellular enzyme activity of cultures from the Eilat, Israel saltern

\section{Molecular analyses}

The relative concentrations of the different operational taxonomic units are displayed in Figure 3. Two OTU's were routinely encountered with base pairs length of 245 and 246. Although these were found during each sampling regardless of salinity, the relative ratios changed over time, Figure 3, even within the same salinity pond. The OTU's do not represent individual organisms but are a composite of all of the microbial community that reacted during the PCR. Therefore, it is not possible to state that any one genus is dominant as each OTU could be a single genus or many genera. As with all PCR-based studies, there is a selectivity in the primers as well as biasis, inhibition due to hairpins, etc. (Litchfield et al., 2006). To estimate the generic diversity it is necessary to clone and sequence the PCR products.

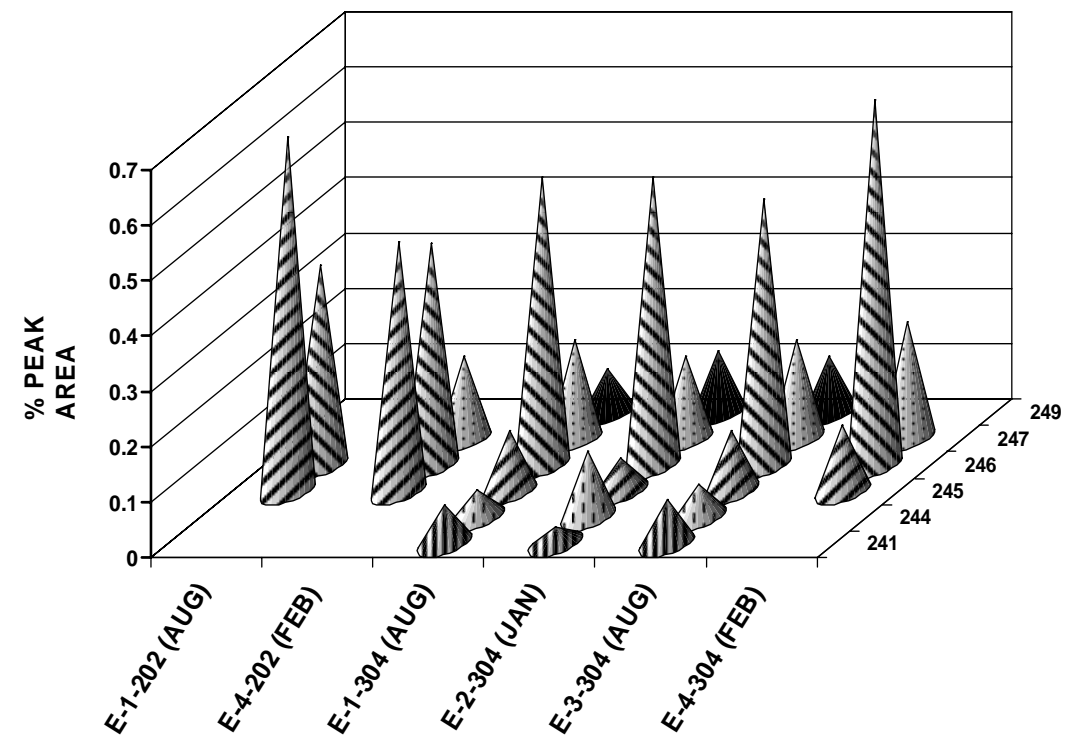

Figure 3. The operational taxonomic units obtained with archaeal primers

\section{CONCLUSIONS}

The greatest diversity occurred a the inlet and lower salinity ponds. This is not surprising as salt in known to select for halotolerant and halophilic microbes. In general, the lower nutrient medium (MR2A) resulted in higher numbers of cultivated microbes. Most of the time the differences between the direct microscopic counts were 1 to 3 orders of magnitude higher than the CFU. This is not surprising in that all media are selective in as much as not all nutrient requirements can be met even with a number of different media and different salinities. 
The metabolic potential of these microbes is extremely variable and probably reflects the diversity of nutrients entering the system. This conclusion is supported by the variety of extracellular enzymes noted for a small sampling of the culture collection from this saltern.

The appearance of two OTU's throughout the study indicates a residual microbial community with an ephemeral one developing alongside depending on the season and salinity of the pond.

Similar results have been observed for other salterns (Rodríguez-Valera et al., 1999; Litchfield et al., 1999; Litchfield et al., 2005) which are less eutrophic and have greater environmental variability that the saltern at Eilat. Thus, solar salterns, worldwide, are an excellent reservoir of utapped microbial diversity and must be preserved.

\section{ACKNOWLEDGEMENTS}

The authors thank George Stojhovic, George Dimitrov, and Liane Agulto for their technical assistance. This research was partially funded by a US-Israel Binational Science Foundation grant No. 95-00027 and by US National Science Foundation grant No. DEB-99-72093.

\section{REFERENCES}

Litchfield, C.D., Irby, A., and Vreeland, R.H. (1999), The microbial ecology of solar salt plants, In: Microbiology and Biochemistry of Hypersaline Environments, Oren, A. (Ed.), CRC Press, Boca Raton, Florida.

Litchfield C.D. and Oren A., (2001). Polar lipid and pigments as biomarkers for the study of the microbial community structure of solar salterns. Hydrobiologia, 466, 81-89.

Litchfield C.D., Irby A., Kis-Papo T. and Oren A., (2001). Comparative metabolic diversity in two solar salterns. Hydrobiologia, 466, 73-80.

Litchfield C.D., Sikaroodi M. and Gillevet P.M., (2005). The Microbial diversity of a solar saltern on San Francisco Bay, In: Adaptation to Life at High Salt Concentrations in Archaea, Bacteria, and Eukarya, Gunde-Cimerman, N., Oren, A., and Plemenitaš (Eds.), Springer, Netherlands.

Litchfield C.D., Sikaroodi M. and Gillevet P.M., (2006). Characterization of natural communities of halophilic microorganisms, In: Extremophiles - Methods in Microbiology, Vol. 35, Rainey, F. A. and Oren, A. (Eds.), Academic Press.

Rodríguez-Valera, F., Acinas, S. G., and Antón, J. (1999), Contribution of molecular techniques to the study of microbial diversity in hypersaline environments, In: Microbiology and Biochemistry of Hypersaline Environments, Oren, A. (Ed.), CRC Press, Boca Raton, Florida.

Suzuki M., Rappé M.S. and Giovannoni S.J., (1998). Kinetic bias in estimates of coastal picoplankton community structure obtained by measurements of small-subunit $r$ RNA gene PCR amplicon length heterogeneity, Applied and Environmental Microbiology, 64, 4522-4529.

Vreeland R.H., Litchfield C.D., Martin E.L., and Elliot E., (1980). Halomonas elongata: A new genus and species of extremely salt tolerant bacteria. International Journal of Systematic Bacteriology, 30, 485-495.

Zweiful U.L. and Hagstrom A., (1995). Total counts of marine bacteria include a large fraction of non-nucleoid-containing bacteria. Applied and Environmental Microbiology, 61, 2180-2185. 